\title{
An orientation-based pruning tool to speed up contact determination between translating polyhedral models*
}

\author{
P. Jiménez $\quad$ C. Torras \\ Institut de Robòtica i Informàtica Industrial (CSIC - UPC) \\ Llorens i Artigas 4-6, E-08028 Barcelona, Spain \\ e-mail:\{pjimenez,ctorras\}@iri.upc.es
}

\begin{abstract}
Contact determination in terms of edge-face intersection tests permits handling nonconvex polyhedra directly, without decomposing them into convex entities. This saves the decomposition time and avoids having to deal with fictitious features, but requires checking all possible pairings. However, by considering only translations and departing from a noninterfering situation, the number of pairings to be checked decreases drastically. The set of critical pairings can be determined efficiently by using the Spherical Face Orientation Graph (SFOG), a representation developed by the authors. An algorithm to exploit the SFOG in convex settings provides controlled evidence of the pruning potential of this approach: the number of critical pairings grows linearly with the complexity of the polyhedra, instead of quadratically as the total number of pairings does. Experiments with a similar algorithm on nonconvex settings confirm the expected potential of the approach: for workpieces with many concavities moving in close proximity, our contact determination procedure performs one order of magnitude faster than RAPID, at the expense of a much higher preprocessing time.
\end{abstract}

\section{INTRODUCTION}

Most collision detection methods have been devised for complex graphic environments, where speed is the main priority. Such environments contain typically a large collection of objects, modeled as convex polyhedra or polygon soups, and the goal is to determine when and where interference may occur. To this end, many techniques to encapsulate possible collisions within time and space bounds have been developed [Jiménez et al., 2001, Lin and Gottschalk, 1998], such as enclosing boxes [Cohen et al., 1995, García-Alonso et al., 1994], object partitioning for CSG representations [Cameron, 1991], space partitioning [García-Alonso et al., 1994, Thibault and Naylor, 1987], hierarchies of bounding volumes [Gottschalk et al., 1996, Hamlin et al., 1992, Hubbard, 1993, Hudson et al., 1997, Klosowski et al., 1998, Martínez et al., 1998, van der Bergen, 1997], space and time coherence [Lin and Canny, 1991, Ponamgi et al., 1997], four-dimensional space-time bounds [Hubbard, 1995] and distance bounding [Gilbert et al., 1988].

This paper deals with an instance of the collision detection problem that has received less attention, namely exact contact determination between two translating nonconvex polyhedra that are in close proximity and which can make contact over wide, possibly disconnected, portions of their boundaries. This situation arises in assembly design and planning within CAD/CAM systems [Thomas and Torras, 1992]. In a previous paper, we tackled the combinatorial search needed to generate optimal $k$-directional translational assembly sequences [Jiménez and Torras, 2000], and here we address the complementary problem of contact determination for translational assembly. In this context, it is crucial to discard features in the two polyhedra that can never get in contact. Back-face culling [Vanecek, 1994] is an effective such pruning strategy based on the direction of motion. Here we propose a related strategy based on the relative orientation of the polyhedra.

The paper is structured as follows. For self-containment, Sections 2 and 3 review the items on which the present work is based: the edge-face intersection test, which permits a decomposition-free contact determination between nonconvex polyhedra [Thomas and Torras, 1994], and the applicability conditions [Donald, 1987] used here to reduce the search for interfering features under arbitrary translational motion.

${ }^{*}$ This paper provides a revised and extended account of results presented at the IEEE Int. Conf. on Robotics and Automation [Jiménez and Torras, 1996, Jiménez and Torras, 1999]. 
these conditions. This representation is exploited in Sections 5 and 6 to speed up contact determination in convex and nonconvex settings, respectively. Section 7 presents experimental results and Section 8 draws some conclusions.

\section{THE EDGE-FACE INTERSECTION TEST}

The boundaries of two polyhedra interfere if and only if at least one edge of one polyhedron intersects a face of the other one. Most contact and interference detection procedures require faces to be convex or triangulated. Others, like the classical crossings algorithm [Boyse, 1979], work on nonconvex faces, but require auxiliary geometric constructs, like shooting a ray from the point of intersection and counting the number of crossings with the boundary edges.

The edge-face intersection test that we will use [Thomas and Torras, 1994] works on the vertices of nonconvex faces directly, without introducing any auxiliary entity. This test is a combination of the two basic contact predicates (vertex-face and edge-edge), which correspond to the truth value (the sign) of two types of functions, computed as $4 \mathrm{x} 4$ determinants of homogeneous vertex coordinates. A vertex $v$ face $f$ predicate $\mathbf{A}_{v, f}$ is true if $A_{v, f}=\left\|v_{i} v_{j} v_{k} v\right\|>0$, where $\left\{v_{i}, v_{j}, v_{k}\right\}$ is an ordered set of vertices of $f$, and an edge-edge predicate $\mathbf{B}_{e_{m}, e_{n}}$ is true if $B_{e_{m}, e_{n}}=\left\|v_{i} v_{j} v_{k} v_{l}\right\|>0$, where $v_{i}=\partial^{+} e_{m}, v_{j}=\partial^{-} e_{m}$, $v_{k}=\partial^{+} e_{n}$, and $v_{l}=\partial^{-} e_{n}\left(\partial^{+}\right.$and $\partial^{-}$are the halfboundary operators - in this case, they refer to the endpoints of the edges).

These functions (and their associated predicates) express incidence relationships between the primitives involved. $A_{v, f}=0$ means that vertex $v$ lies on the plane that supports $f$, and the sign of this function, when different from zero, indicates on which of the two halfspaces defined by this plane $v$ lies. $B_{e_{m}, e_{n}}=0$ means that the lines supporting the edges are either touching or parallel, other relative positions and orientations are expressed through the sign of this function.

Now, for an edge $e$ to intersect a possibly nonconvex face $f$, two conditions must simultaneously hold:

- the two endpoints of the edge must lie in opposite halfspaces of those defined by the plane supporting the face, and

- the line supporting the edge must intersect the face.

The edge-face test checks these two conditions by evaluating the following composite predicate:

$$
\left(\mathbf{A}_{\partial^{+} e, f} \oplus \mathbf{A}_{\partial^{-} e, f}\right) \wedge\left[\bigoplus_{e_{f} \in \partial f}\left(\mathbf{A}_{\partial^{+} e_{f}, f_{e}} \oplus \mathbf{A}_{\partial^{-} e_{f}, f_{e}}\right) \wedge\left(\mathbf{A}_{\partial^{-} e_{f}, f_{e}} \oplus \mathbf{B}_{e, e_{f}}\right)\right]
$$

where $\oplus$ is the XOR exclusive OR operator $((a \oplus b)=(a \wedge \bar{b}) \vee(\bar{a} \wedge b))$, and $f_{e}$ refers to any plane containing edge $e$. To identify the right term with the second condition, note that the number of edges in the boundary of $f$ that pierce one of the halfplanes of $f_{e}$ must be odd for the condition to hold. See Figure 1 and [Thomas and Torras, 1994] for further details. The predicate can be seen as an extension of Canny's disjunctive form [Canny, 1987] to deal with nonconvex faces.

In order to test for interference between polyhedra, the above predicate has to be applied to the pairings consisting of edges of one polyhedron and faces of the other one. It suffices to determine one such pairing for which the predicate holds to report interference. But this search has a quadratic worstcase complexity: if no interference exists, all edge-face pairings have to be tested. Therefore, any technique that lowers the number of edge-face pairings to be considered will be welcome. This can be done if one assumes that the polyhedra are initially disjoint and translating, and that only the first edge-face pairing that may intersect needs to be reported.

\section{APPlicability CONDItions AND THEIR USE FOR PRUNING EDGE- FACE PAIRS}

Consider two disjoint polyhedra. If any relative motion between them is allowed, many contacts between the features of both polyhedra are possible (in particular, when the polyhedra are convex, every contact is possible). But if only translations are allowed, only certain contacts can arise. These contacts are said to be applicable. In the case of convex polyhedra, necessary and sufficient conditions for vertex-face and edge-edge applicability have been stated [Donald, 1987] (refer to Figure 2). For a given relative orientation between two polyhedra: 


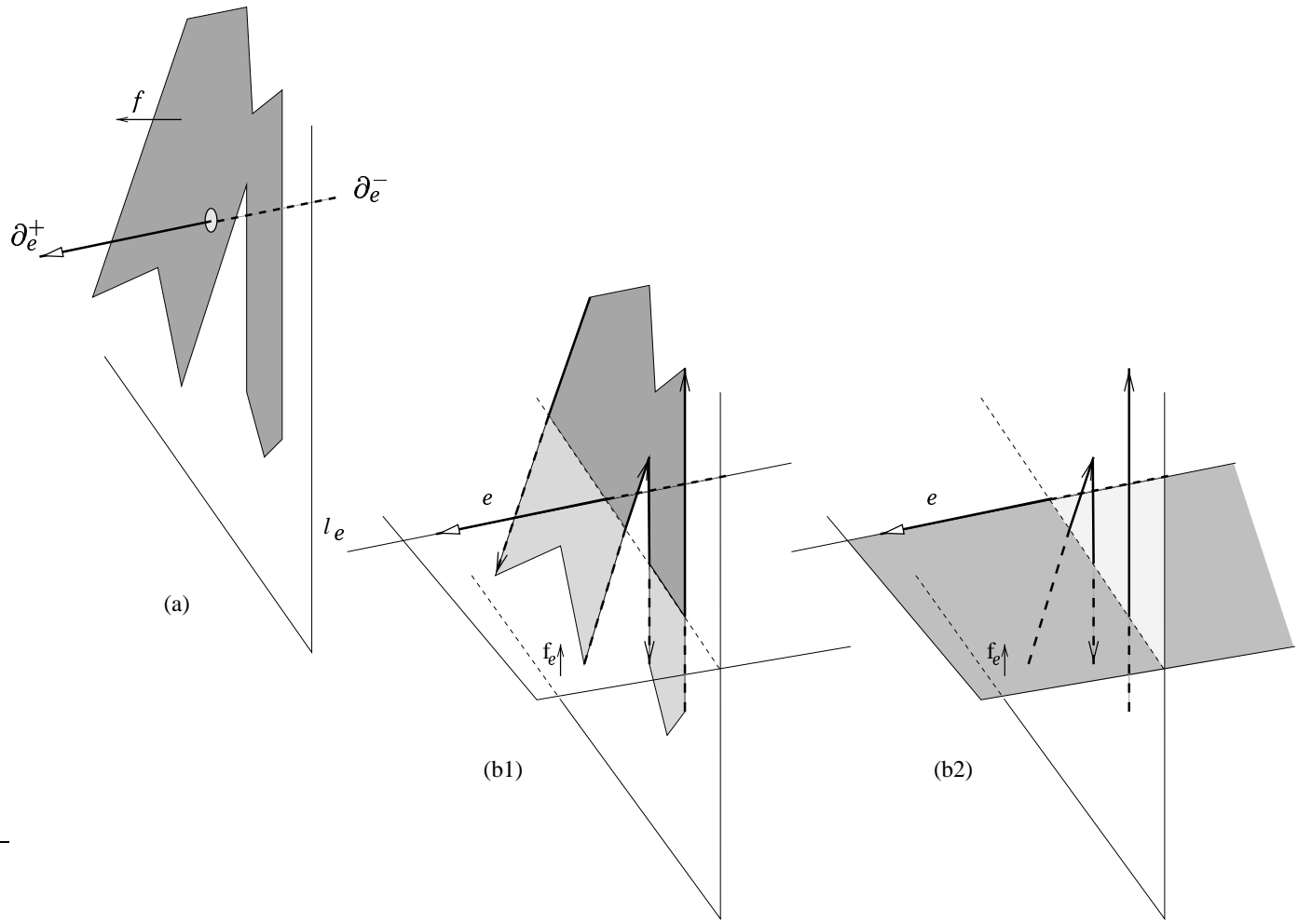

Figure 1: (a) $\mathbf{A}_{\partial^{+}, e, f} \oplus \mathbf{A}_{\partial^{-}, \text {e,f }}$ is true if and only if both endpoints of edge $e$ lie on different sides of the supporting plane of face $f$. (b) Testing whether the line $l_{e}$ supporting e cuts $f$ is done by (b1) determining the set of boundary edges of $f$ that intersect the arbitrary plane $f_{e}, \mathbf{A}_{\partial+e_{f}, f_{e}} \oplus \mathbf{A}_{\partial^{-}} e_{f}, f_{e}$, and (b2) counting the number of these edges that intersect the plane to the right (or to the left) of $l_{e}$. This number is odd (the $X O R$ operator is used for parity testing) if and only if the second condition of the edge - face intersection test holds. 
$\forall v_{i}$ adjacent to $v,\left\langle v_{i}, f\right\rangle-\langle v, f\rangle \geq 0$

- the contact between an edge $e_{m}$ of one polyhedron and an edge $e_{n}$ of another polyhedron is applicable iff $k_{a} \neq k_{b}$, where $k_{a}=\operatorname{sign}\left(\left\langle T_{1}, f_{p}\right\rangle\right)=\operatorname{sign}\left(\left\langle T_{2}, f_{p}\right\rangle\right)$, and $k_{b}=\operatorname{sign}\left(\left\langle T_{3}, f_{p}\right\rangle\right)=$ $\operatorname{sign}\left(\left\langle T_{4}, f_{p}\right\rangle\right)$, with $f_{p}=e_{m} \times e_{n}$ (or the opposite direction, the choice is arbitrary) and $T_{i}=$ $s_{i} \cdot\left(f_{i} \times e_{m}\right), f_{i}$ adjacent to $e_{m}, T_{j}=s_{j} \cdot\left(f_{j} \times e_{n}\right), f_{j}$ adjacent to $e_{n} ; s_{i}, s_{j} \in\{+1,-1\}$ such that $T_{l}$ is oriented towards the interior of face $f_{l}$.

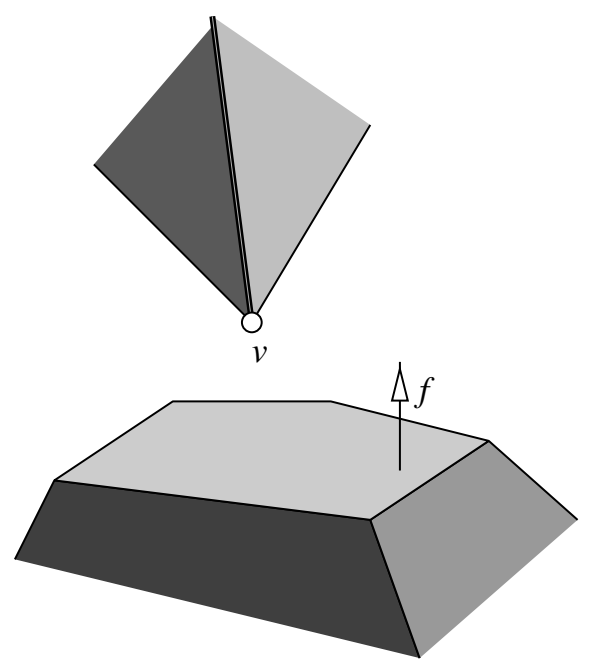

(a)

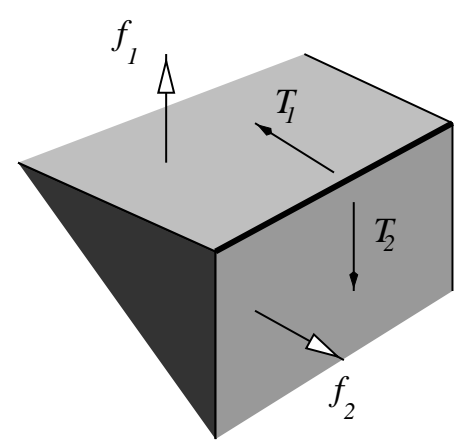

(b)

Figure 2: (a) Applicable vertex - face contact. (b) Applicable edge - edge contact.

If the polyhedra are initially disjoint and translating, the interference test in the preceding section needs to be applied only to the edge-face pairings obtained from the applicability conditions as follows:

- From the vertex-face contact, any edge stemming from the vertex should be paired with the face.

- From the edge-edge contact, any face adjacent to an edge should be paired with the other edge.

Note that, although some repeated pairings may be obtained, both types of applicability need to be considered, since otherwise some candidate pairings could be missed, as depicted in Figure 3.

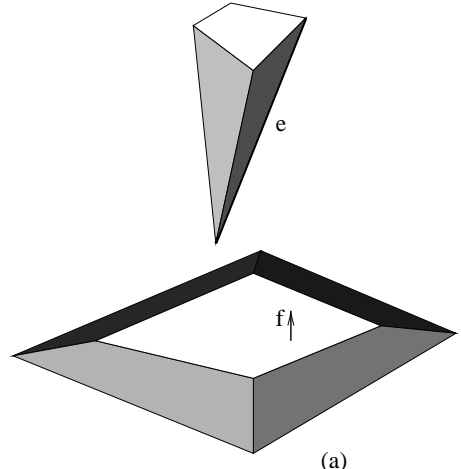

(a)

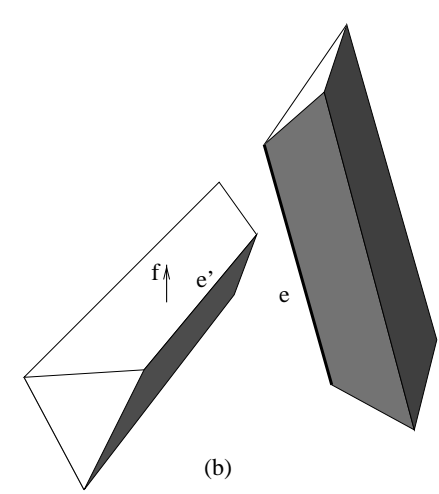

(b)

Figure 3: (a) The candidate pairing $e-f$ can only be obtained from the applicable $v-f$ pair, as no edge in the boundary of $f$ can contact the edges stemming from the vertex. (b) The candidate $e-f$ can only come from the applicable $e-e^{\prime}$ pair, as no endpoint of either edge can contact the faces adjacent to the other edge.

In the convex case, the applicability conditions guarantee that the contact is possible. If the polyhedra are nonconvex, applicability expresses a necessary but not sufficient condition for contact. One has to talk about local applicability in the nonconvex case, as far as only the adjacent features are considered 
being actually possible. Therefore, a distinction has to be made between (locally) applicable contacts and those which are also realizable. Figure 4 shows a locally applicable contact which is not realizable. Edge-face pairings arising from such a situation will be called false candidates.
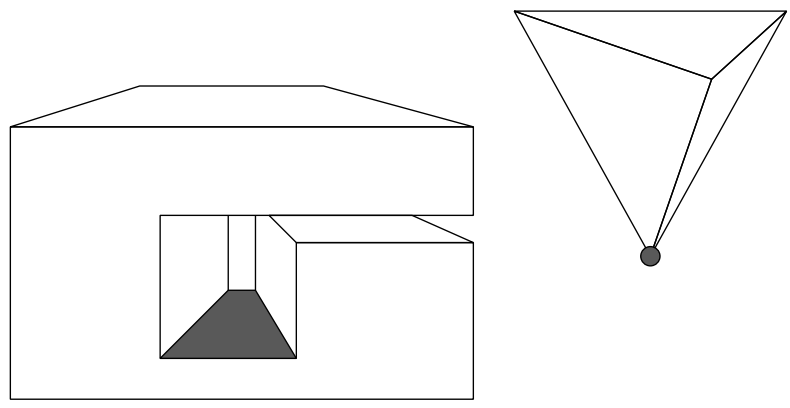

Figure 4: A locally applicable but not realizable vertex - face contact.

\section{A suitable Representation For DeteCting applicable CON- TACTS}

\subsection{The Spherical Face Orientation Graph (SFOG)}

As shown in the preceding section, the applicability of contacts between surface features depends on the relative orientations of these features. Thus, a natural frame for representing applicability is the unit sphere of orientations. The Gaussian map of a given surface represents the surface normals as points on the unit sphere [Hilbert and S.Cohn-Vossen, 1987, Horn, 1984]. The SFOG extends this concept by representing also edges and vertices of polyhedra, and their adjacencies, as described next.

Faces are represented by nodes on this sphere. In fact, the node represents the orientation of the outward normal of the plane supporting the face, i.e. that pointing outside the polyhedron.

Edges are represented by arcs. These arcs join nodes that correspond to faces sharing an edge. Geometric consistency is attained by placing these arcs on great circles of the sphere. In this way, the normals of the planes that define these great circles point in the same directions as the corresponding edges. Convex edges are represented by means of the minor arc, concave edges with the major arc. The representation, thus, characterizes the type of arc and is coherent with the criterion of considering the supplementary angle of the internal dihedrical angle between the faces. The arcs will be called convex or concave depending on the type of edge they represent.

A vertex is represented by the region enclosed within a cycle of convex arcs and nodes, corresponding to adjacent edges and faces. This region is well defined for convex vertices (where all the adjacent edges are convex). Nonconvex vertices have one or more nonconvex adjacent edges. For some nonconvex vertices (called here pseudo-convex) it is possible to select a subset of adjacent convex edges that define locally a pyramid which contains all other adjacent edges (this pyramid is the local convex hull of the vertex). The convex arcs that correspond to these edges enclose a so-called convex subregion (csr) on the sphere

Figure 5 shows how the three types of features of a polyhedron are represented on the SFOG, for a convex vertex, and Figure 6 illustrates the concepts of convex subregion and local convex hull for a pseudo-convex vertex.

Correctness of this representation is proven in [Jiménez, 1998].

The SFOG is not unambiguous: a polyhedron may not be reconstructed from this representation, neither in size nor completely in shape, as shown in Figure 7. Nevertheless, although this ambiguity may be a drawback for certain applications, it is not a problem for the purposes pursued here. The point is that the SFOG representation preserves those geometric relations that are relevant for the applicability conditions.

\subsection{Pairing of applicable features using the SFOG representation}

By superimposing the SFOG of one polyhedron on the central symmetric image of the SFOG of another polyhedron (see Figure 8), a compact representation is obtained from which the vertex-face and edge-edge applicability relationships can be directly determined: 

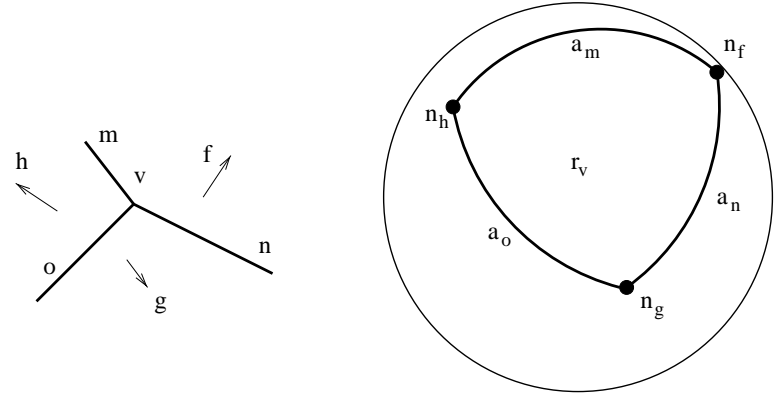

Figure 5: Representation of polyhedral features on the SFOG: a vertex $v$ is represented by region $r_{v}$, its adjacent faces $f, g$ and $h$ by the nodes $n_{f}, n_{g}$ and $n_{h}$, and the adjacent edges $m, n$ and $o$ by the arcs $a_{m}$, $a_{n}$ and $a_{o}$.

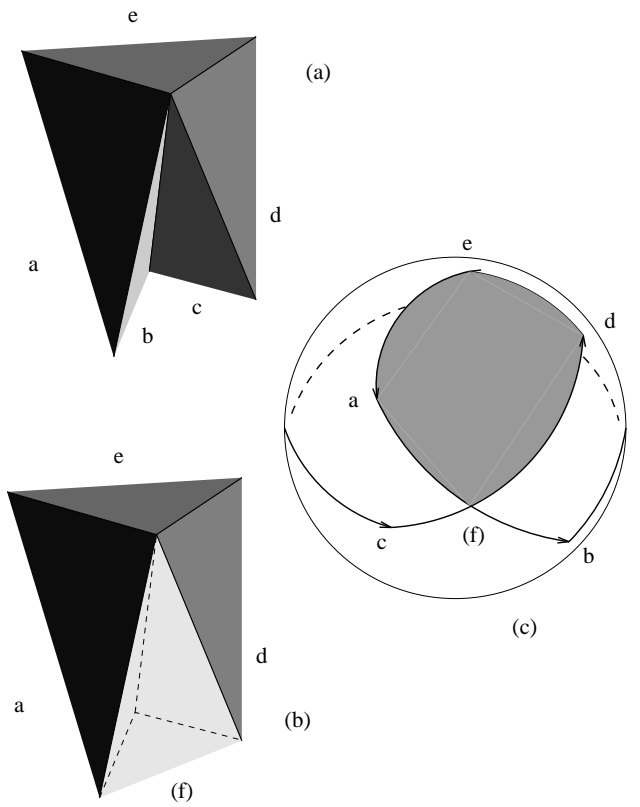

Figure 6: (a) A pseudo-convex vertex, (b) its local convex hull, and (c) the corresponding csr.
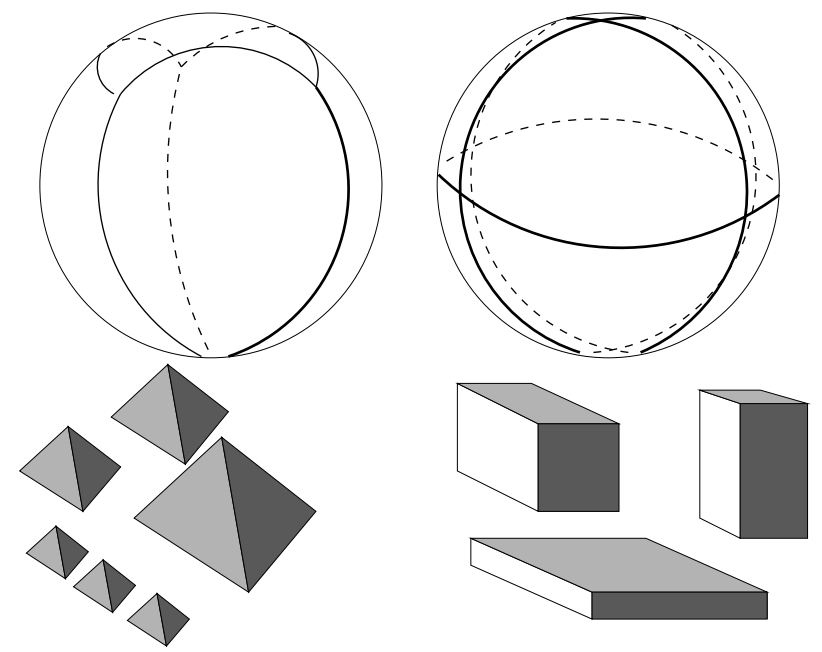

Figure 7: Different polyhedra will have the same representation on the SFOG if the relative orientations of the faces and adjacency relationships are preserved. 
between the vertex represented by the region and the face represented by the node is applicable (Figure 9).

2. (Nonconvex vertex-face applicability) A given node falls into a certain convex subregion ( $c s r$ ) if and only if the contact between the vertex whose local convex hull is represented by the csr and the face represented by the node is locally applicable (Figure 10).

3. (Edge-edge applicability) Two convex arcs of different SFOGs intersect if and only if the contact between the corresponding edges is (locally, in the nonconvex case) applicable (Figure 11).

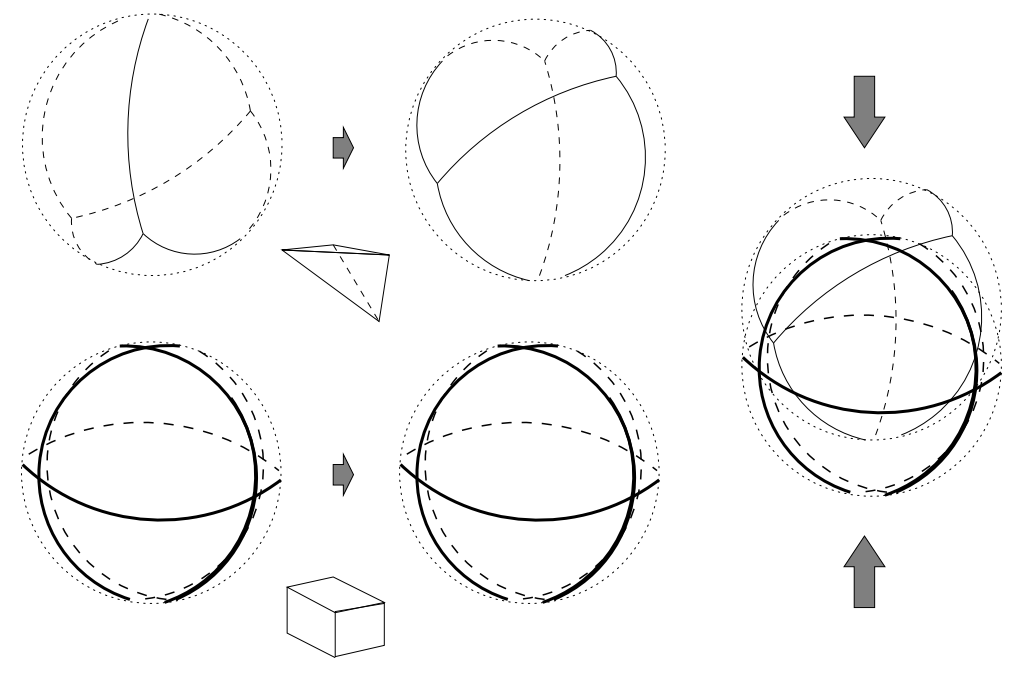

Figure 8: Overlay of the SFOGs corresponding to two polyhedra in order to obtain a compact representation that allows to determine the applicability relationships. The SFOG of the rectangular prism below (heavy lines) is combined with the central symmetric image of the SFOG of the tetrahedron above (fine lines).

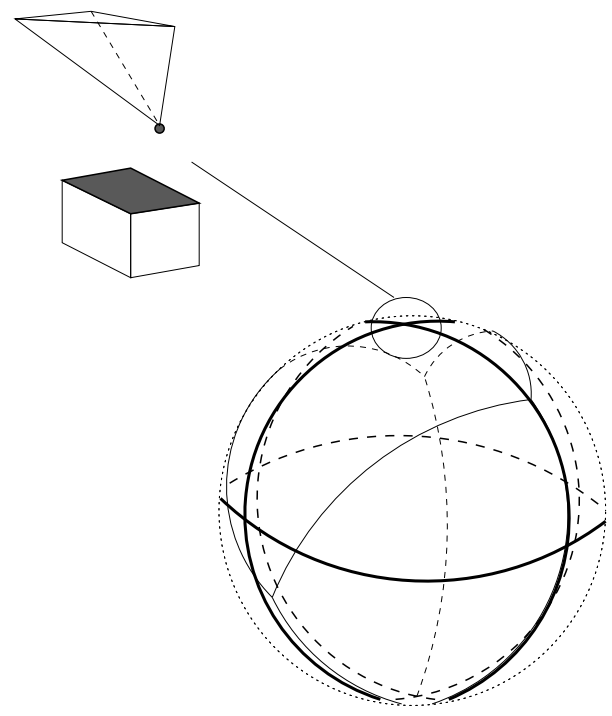

Figure 9: An applicable vertex-face contact, where the vertex is convex.

The representation is consistent with all the facts concerning applicability, as the existence of at least one applicable vertex per face (each node is inside of at least one region), or the possibility of nonexistence of an applicable face for a given vertex (a region may have no nodes inside), among others. An important difference between the SFOGs of convex and nonconvex polyhedra has to be stressed: in the convex case, the regions corresponding to the vertices constitute a partition of the spherical surface, whereas for nonconvex polyhedra the convex subregions cover the sphere and may overlap. This means 


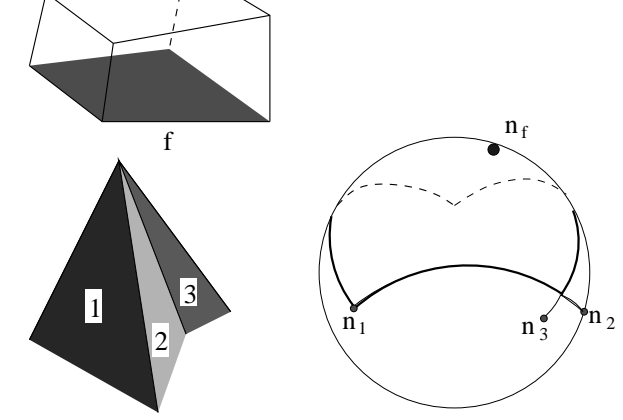

Figure 10: An applicable vertex-face contact, where the vertex $v$ is pseudo-convex. The node $n_{f}$ corresponding to the face $f$ is contained in the convex subregion corresponding to the local convex hull of the vertex.

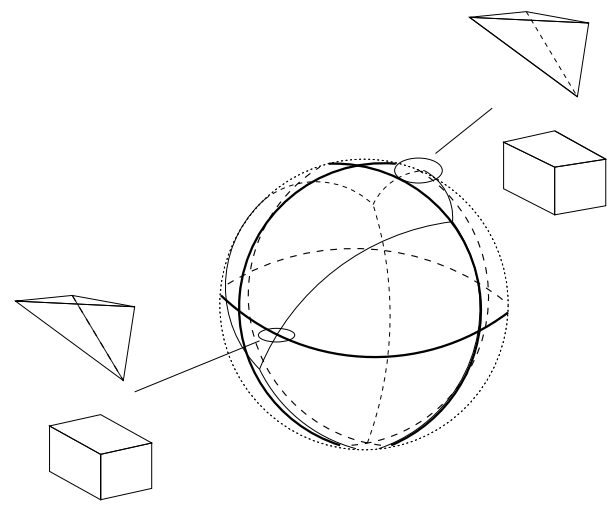

Figure 11: Two examples of applicable contact between edges. The corresponding arcs intersect.

that in the latter case, a given face may be simultaneously applicable to various vertices, if the node that represents this face lies inside the intersection of the corresponding convex subregions. Figure 12 shows one such situation.
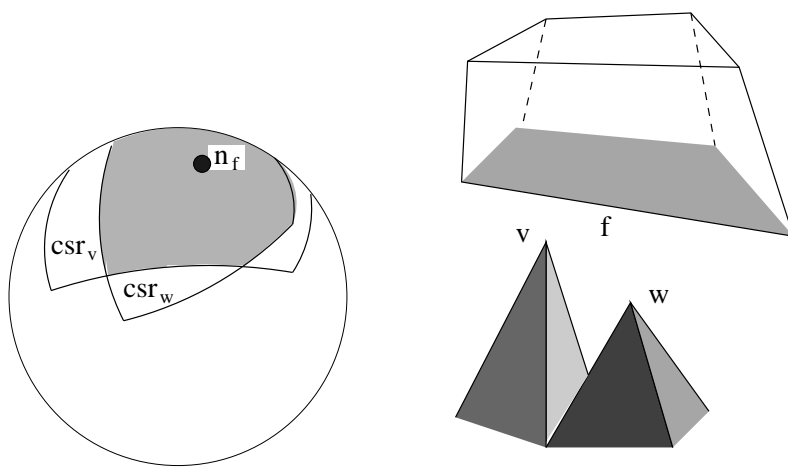

Figure 12: For nonconvex polyhedra, situations may exist where the same face is simultaneously applicable to several vertices: the corresponding node is contained in the common area of the overlapping csr's of these vertices.

The next step is to develop an algorithm to obtain all the applicable feature pairs efficiently.

\section{THE CONVEX CASE}

The amount of pruning that can be done becomes particularly evident in the convex case. Therefore, a simple algorithm has been developed and implemented for the situations where the polyhedra are known to be convex. This algorithm considers nodes of one SFOG and regions of the other one, and performs the node-in-region inclusion and the arc crossings tests. 
- input graphs:

- the SFOGs

- a Cycle Graph (where nodes correspond to vertices of one polyhedron)

- output vectors:

- FACE_APP $[<$ node $>$ ] recording the face-vertex applicability relationships

- EDGE_APP $[<\operatorname{arc}>$ ] recording the edge-edge applicability relationships

- processing lists:

- OPEN_NODES

- OPEN_ARCS

A brief description of procedures and functions is needed for the clear understanding of the algorithm. The procedure ordered_intersection (node, $\mathscr{G} E D G E \_A P P$ ) finds every intersection of arcs stemming from node with the arcs of the cycle in which node lies, and appends these intersections to EGDE_APP. The arc_intersection(arc, cycle, edge) function finds the intersection between arc and the arcs of cycle different from edge, which is known to have been crossed by arc in entering cycle. The function Succ_Arcs(node) returns the arcs that "point out of" node, in the sense that although we are exploring an undirected graph, certain directions of the arcs are implicitly imposed as some nodes are explored before others and we want to avoid exploring a given arc in both directions. The function succ_node(arc) returns the unexplored extreme node of arc, and succ_cycle(edge, arc) returns the cycle that cobounds edge and where arc is "pointing to" (in the sense that the other cobounding cycle will either contain the node such that arc $\in$ Succ_Arcs(node) or will already have an arc intersected by $\operatorname{arc}$ ). Finally, the function last(EDGE_APP [arc]) returns the last arc intersected by arc.

The algorithm starts at a given point, which can be considered without loss of generality as a "North Pole", and travels over the sphere towards the "South" in a spiral-like fashion. Thus, it can be considered a greedy or breadth-first algorithm [Pearl, 1984].

\section{SFOG SEARCH ALGORITHM (convex polyhedra)}

Choose North-pole;

Find North-region $\supset$ North-pole;

FACE_APP[North-pole]:= North-region;

North-pole $\rightarrow$ OPEN_NODES;

$\underline{\text { while }}\left(O P E N_{-} N O D E S \neq \emptyset\right)$ or $\left(O P E N_{\_} A R C S \neq \emptyset\right)$

$\underline{\text { while }}\left(O P E N_{-} N O D E S \neq \emptyset\right)$

node $\leftarrow$ OPEN_NODES;

ordered_intersection(node, $\left.8 E D G E \_A P P\right)$;

for every $a \in$ Succ_Arcs(node)

if $E D G E \_A P P[a]=\emptyset \underline{\text { then }}$

if FACE_APP[succ_node $(a)]=\emptyset \underline{\text { then }}$

FACE_APP[succ_node $(a)]:=$

FACE_APP[node];

succ_node $(a) \rightarrow$ OPEN_NODES;

endif

$\underline{\text { else }}$

$a \rightarrow$ OPEN_ARCS;

$\frac{\text { endif }}{\text { endfor }}$

$\underline{\text { endwhile }}$

$\underline{\text { while }}\left(O P E N_{-} A R C S \neq \emptyset\right)$

arc $\leftarrow$ OPEN_ARCS;

edge $:=$ last $\left(E D G E \_A P P[\operatorname{arc}]\right)$;

cycle $:=$ succ_cycle (edge, arc);

$s:=\operatorname{arc\_ intersection(arc,~cycle,~edge);~}$ 
endpoint of an arc is reached, that arc is deleted from the active list. In this way, every purple intersection will be detected exactly once. A pseudo-code transcription of this simple algorithm is presented:

\section{NAÏVE SFOG SEARCH ALGORITHM (nonconvex polyhedra)}

Preprocessing: Order the $2 n$ endpoints e[i] by increasing meridian value. Let a[i] be the arc with endpoint e[i] and $c[i]$ its color.

$\underline{\text { for }}(i=1 . .2 n) \underline{d o}$

if (e[i] is a western endpoint) then

$\underline{\text { for }}\left(\right.$ all $\left.a^{\prime} \in L_{\bar{c}[i]}\right) \underline{d o}$

$$
\text { if }\left(a[i] \cap a^{\prime}\right) \underline{\text { then }}
$$

report purple intersection

$$
\frac{\text { endfor }}{\text { insert }}\left(a[i], L_{c[i]}\right)
$$

$\underline{\text { else }}$

$$
\operatorname{delete}\left(a[i], L_{c[i]}\right)
$$

endfor

$$
\frac{\text { endif }}{r}
$$

As said before, the "first" endpoint is an arbitrary choice and, at this first instant, no lists of active arcs exist. Therefore, a second sweep will have to be performed to take into account all the purple intersections with arcs that are still active after the last endpoint. Figure 14 depicts the purple intersections that can be detected along the first sweep and those which cannot be determined if no second sweep is performed.

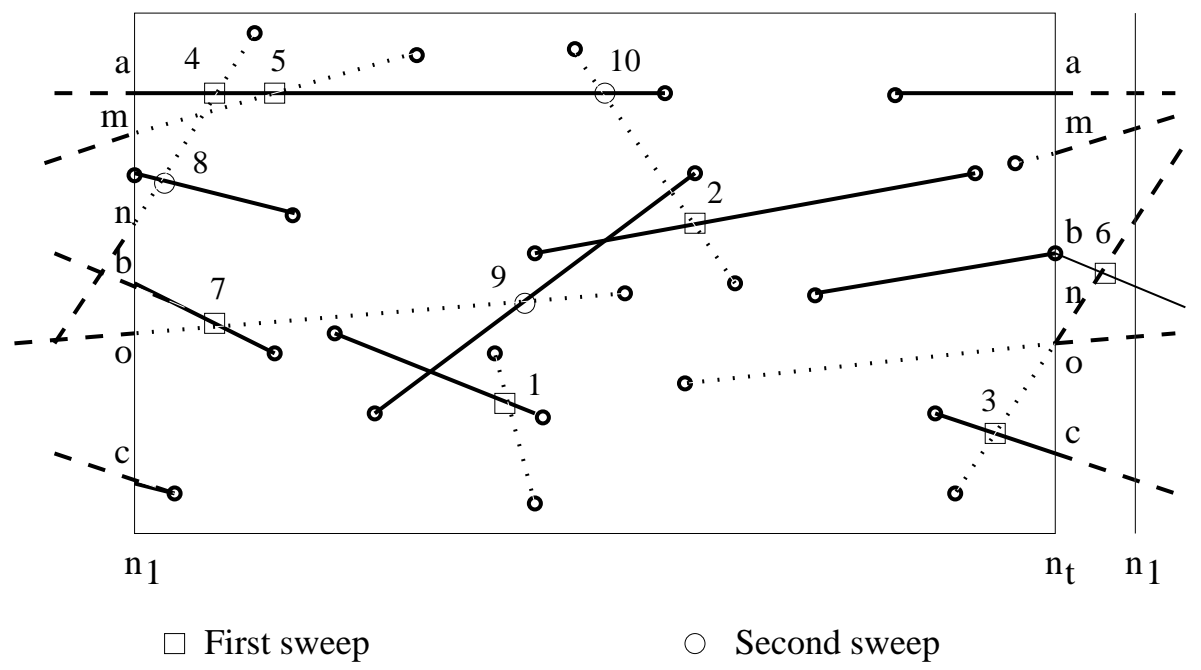

Figure 14: Plane analogue of the spherical sweep. Red and blue arcs are depicted as dotted and solid segments. Some of them $(a, b, c, m, n, o)$ begin before or at the last event point $n_{t}$ and end after the first one $n_{1}$. These segments are activated during the first sweep from e[1] to e[2n], where some purple intersections can already be detected (marked with a small square), and their intersections with the segments that had ended before they where generated (marked with an empty circle) can only be detected during a second sweep. Numbers indicate the order in which the intersections are computed.

As for node-in-region inclusions, they are computed during the same sweeping operation: each region defines an interval on the sweep-line and it has to be determined which intervals of the opposite color include the current endpoint. Each interval is defined by the upper and lower arcs bounding the region at every instant. Regions begin and end at given (not necessarily all) endpoints. The regions a, say, red node belongs to are computed by determining the blue arcs that cut the sweep-line above this node and then finding out if the regions underneath these arcs are also bounded by arcs that cut the sweep-line below that node.

\section{Experimental results}

Consider the three algorithms: 
Algorithm B consists of the specific SFOG search procedure for convex polyhedra (Section 5), followed by interference tests for the resulting edge - face pairings;

Algorithm C consists of the naïve SFOG search procedure for nonconvex polyhedra (Section 6), followed by interference tests for the resulting edge - face pairings.

For comparison purposes, these three algorithms have been implemented and experiments have been carried out over two sets of non-intersecting pairs of polyhedra, convex and nonconvex. The data structure that stores the polyhedra is basically a geometric description of faces (coordinates of their vertices and supporting planes), and the topological information is contained in an adjacency matrix. This structure captures implicitly the information about the edges, which leads towards certain inefficiency when this information has to be made explicit. Mainly, this means that every edge is counted twice when the whole polyhedron is examined, which could be avoided using another structure like the doubly-connectededge-list (DCEL, [Preparata and Shamos, 1985]). Nonetheless, the results are suitable for comparison purposes, as the same data structure constitutes the input in all three cases.

\subsection{Convex polyhedra}

Despite the fact that general polyhedra (i.e., both convex and nonconvex) are the target of our pruning strategy, we first restrict our testbed to convex solids exclusively. This permits testing the algorithm in Section 5 and assess the pruning potential of the strategy in a controlled setting. Convex polyhedra allow to design situations that cover the range from a high degree of pruning (like in polyhedra that approximate spheres) to almost none (like in prisms of high degree), without the distortions due to false candidates. The convex polyhedra used in the experiments range from the tetrahedron to a polyhedral approximation of the sphere with 128 triangular faces, and include other regular and semiregular polyhedra, pyramids, prisms, etc.

Experiments show that it is worthwhile to perform pruning when the polyhedra become complex, and that the threshold lies at a relatively low level. Results are displayed in Figures 15, 16 and 17 (please note that a logarithmic scale is used). In Figure 15 the number of candidate edge-face pairs before and after pruning are displayed, for different relative orientations of the polyhedra. While this number grows quadratically (linearly in the logarithmic scale) if all possible pairings have to be considered, the savings derived from pruning reduce this growth to a linear one. Execution times in Figure 16 correspond to a SG O2 workstation, $180 \mathrm{Mhz}, 192 \mathrm{MB}$ RAM, SPEC int95 4.8, SPEC fp95 5.4. As can be seen, savings in computational time attain various orders of magnitude. Computation of the applicable feature pairings and pruning needs only to be done once, while the polyhedra maintain their relative orientations. Even if the time to perform these computations is included, the total time is less than that needed by Algorithm A, as the polyhedra become more complex, as shown in Figure 17.

\subsection{Nonconvex polyhedra}

Algorithms $\mathrm{A}$ and $\mathrm{C}$ have been tested on a set of nonconvex polyhedra ranging from a pentahedron (with only one concave edge, i.e., a pyramid with a V-shaped base) to an hour-glass shaped polyhedron (160 edges, including 32 concave ones). The results are displayed below. Figure 18 shows a drastic reduction in the number of edge - face intersection tests to perform for the considered objects. Figure 19 displays the execution times of algorithms $\mathrm{A}$ and $\mathrm{C}$, the latter without and with the preprocessing step. We have to insist that this preprocessing has to be done only once. Once again, a logarithmic scale is used in both figures.

Next, we have carried out some experiments to assess the scalability of the approach, and to compare its performance against that of a well-known collision detection package (RAPID, v2.01, publicly available at http://www.cs.unc.edu/ geom/OBB/OBB.html). As a testbed we have used pairs of cubes having an increasing number of square prismatic holes arranged on square grids, as shown in Figure 20.

The two cubes are located and oriented such that the algorithms are forced to perform a high number of elemental intersection tests (e.g., for the case of both cubes having $12 \times 12$ holes, RAPID reports 22947 contacts, out of 576267 box tests, while algorithm C performs 171400 edge-face intersection tests).

Figure 21 shows the increasing power of applicability pruning as the complexity of the setting grows. Again, a linear growth of the number of edge-face tests to perform is observed, versus the quadratic growth of the total number of edge-face pairings.

The experimental results displayed in Figure 22 show that, for such kind of settings, our algorithm is one order of magnitude faster than RAPID in carrying out contact determination. For example, in 


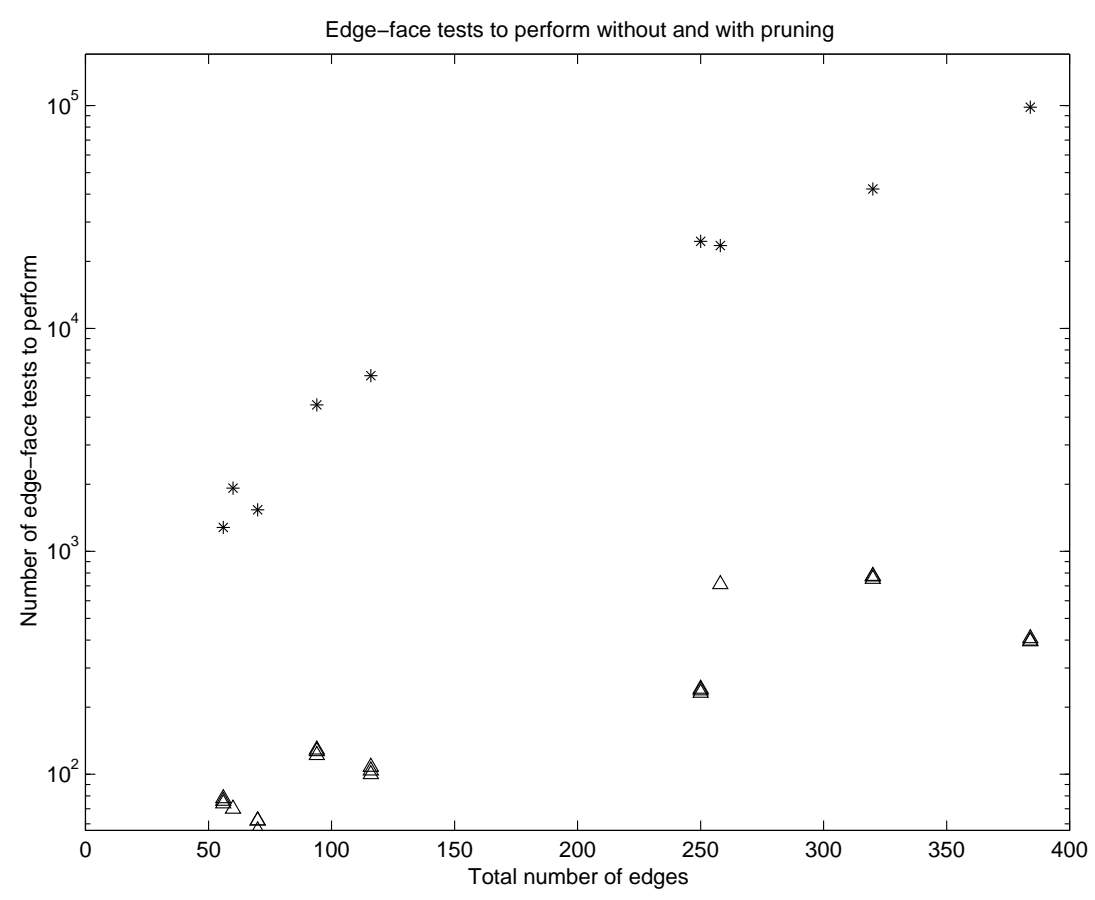

Figure 15: Comparison between the total number of edge - face tests to perform without applicability pruning, i.e. algorithm $A\left({ }^{*}\right)$, and with a previous pruning step, algorithm $B(\triangle)$.

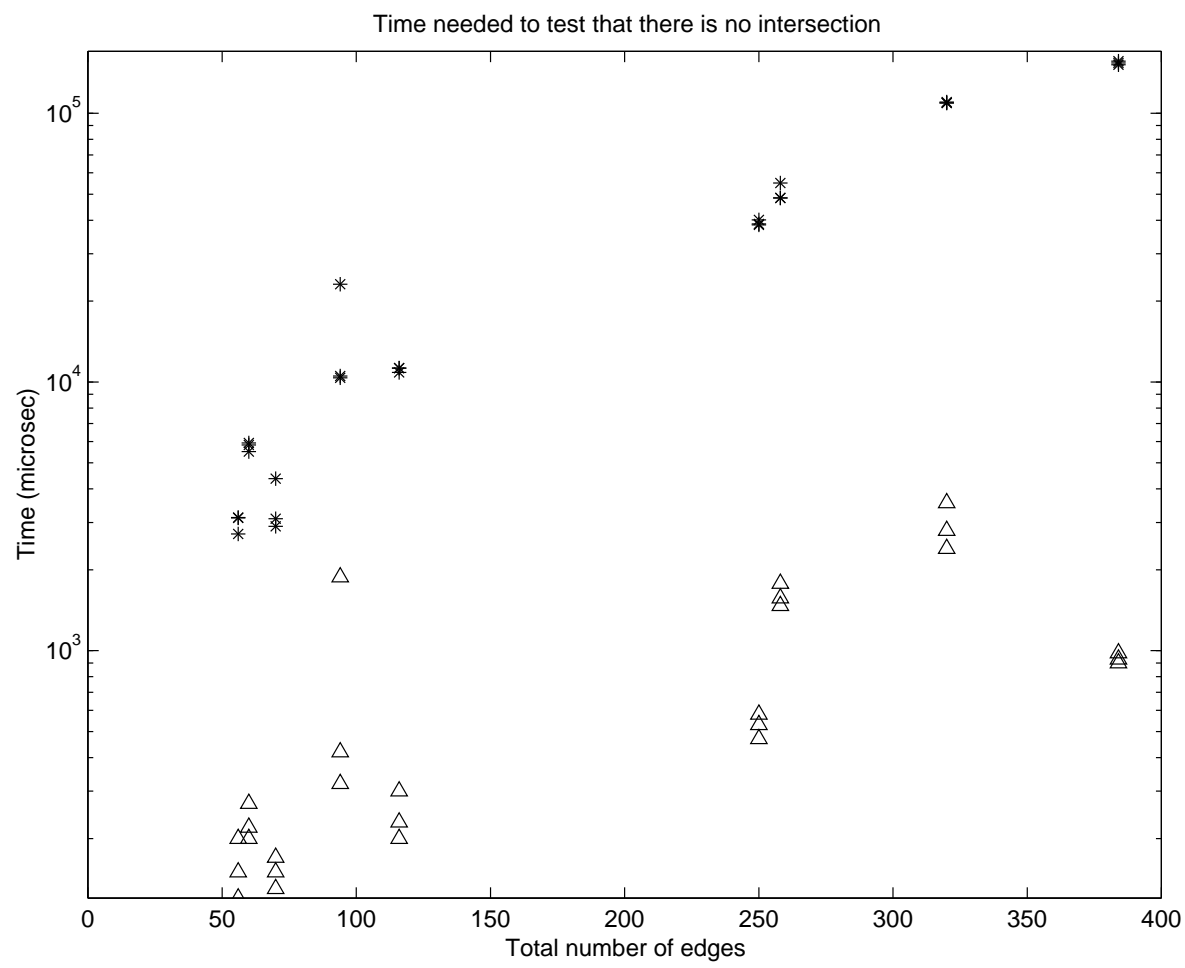

Figure 16: Comparison of execution times of algorithm $A\left({ }^{*}\right)$ and the interference part of $B(\triangle)$. 
Time needed to test that there is no intersection

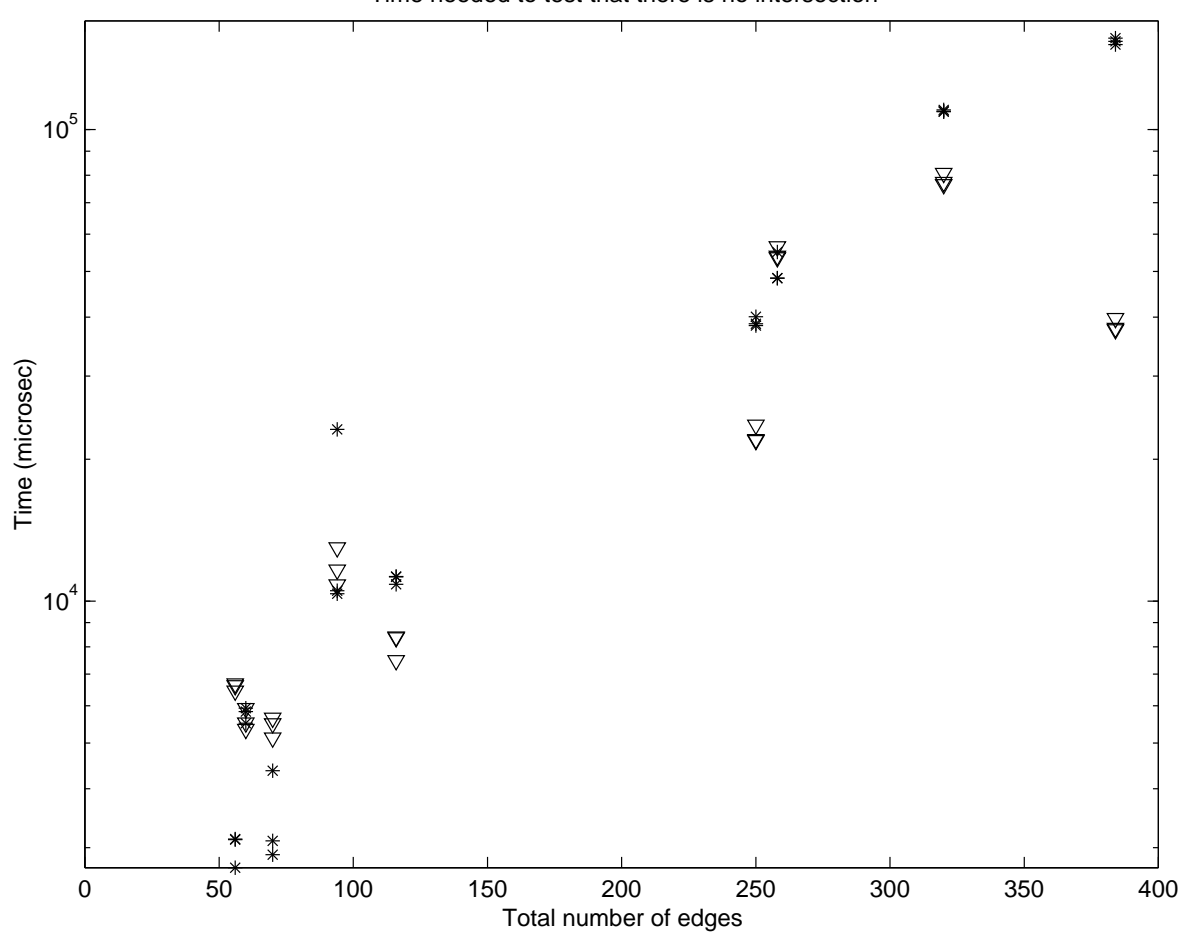

Figure 17: Comparison of execution times of algorithm $A\left({ }^{*}\right)$ and $B$ including preprocessing time $(\nabla)$. Note that with preprocessing added, B outperforms $A$ even for low complexities. The different results for each pair of polyhedra correspond to different relative orientations.

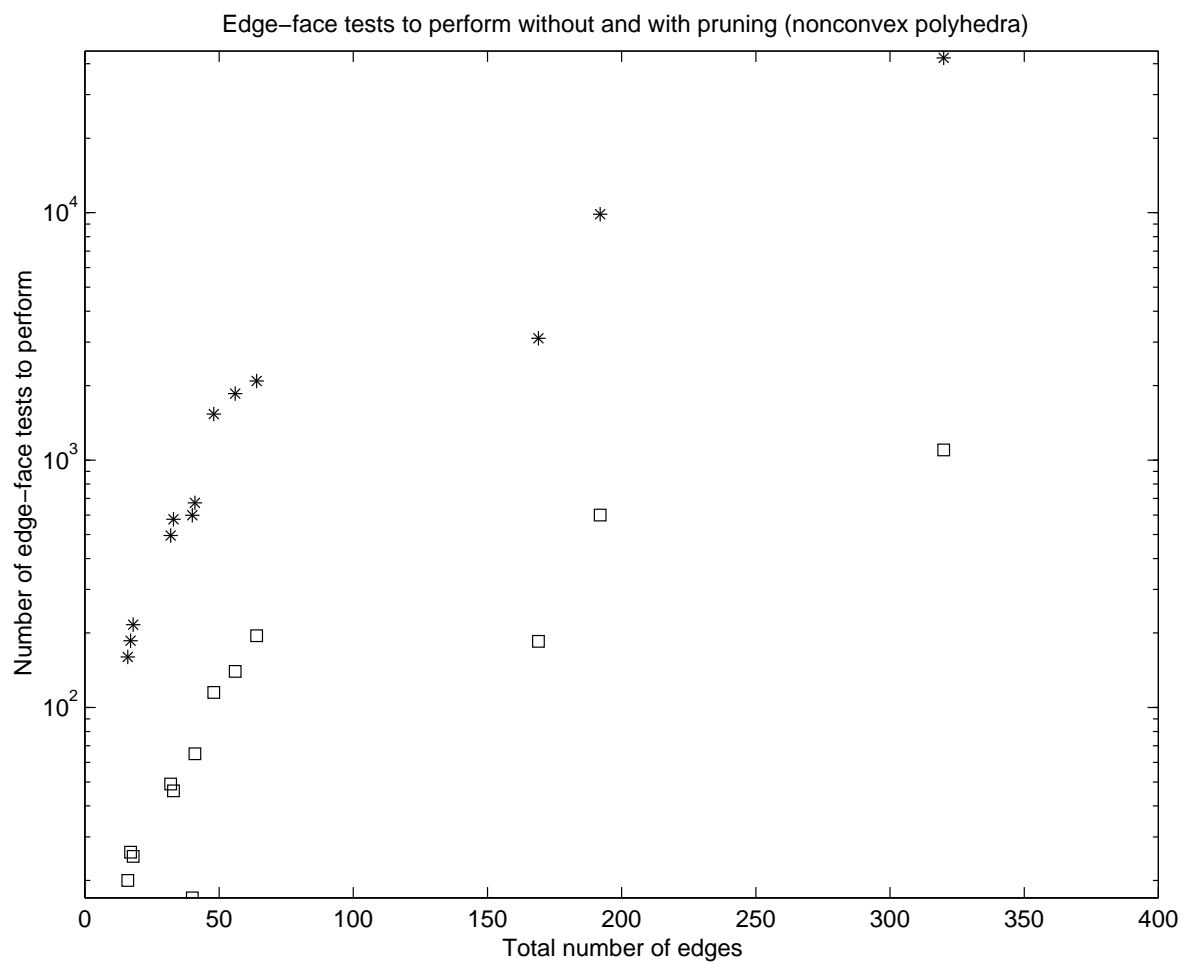

Figure 18: Comparison between the total number of edge - face tests to perform without applicability pruning, i.e. algorithm $A\left({ }^{*}\right)$, and with a previous pruning step, algorithm $C(\square)$, in settings that include only nonconvex polyhedra. 


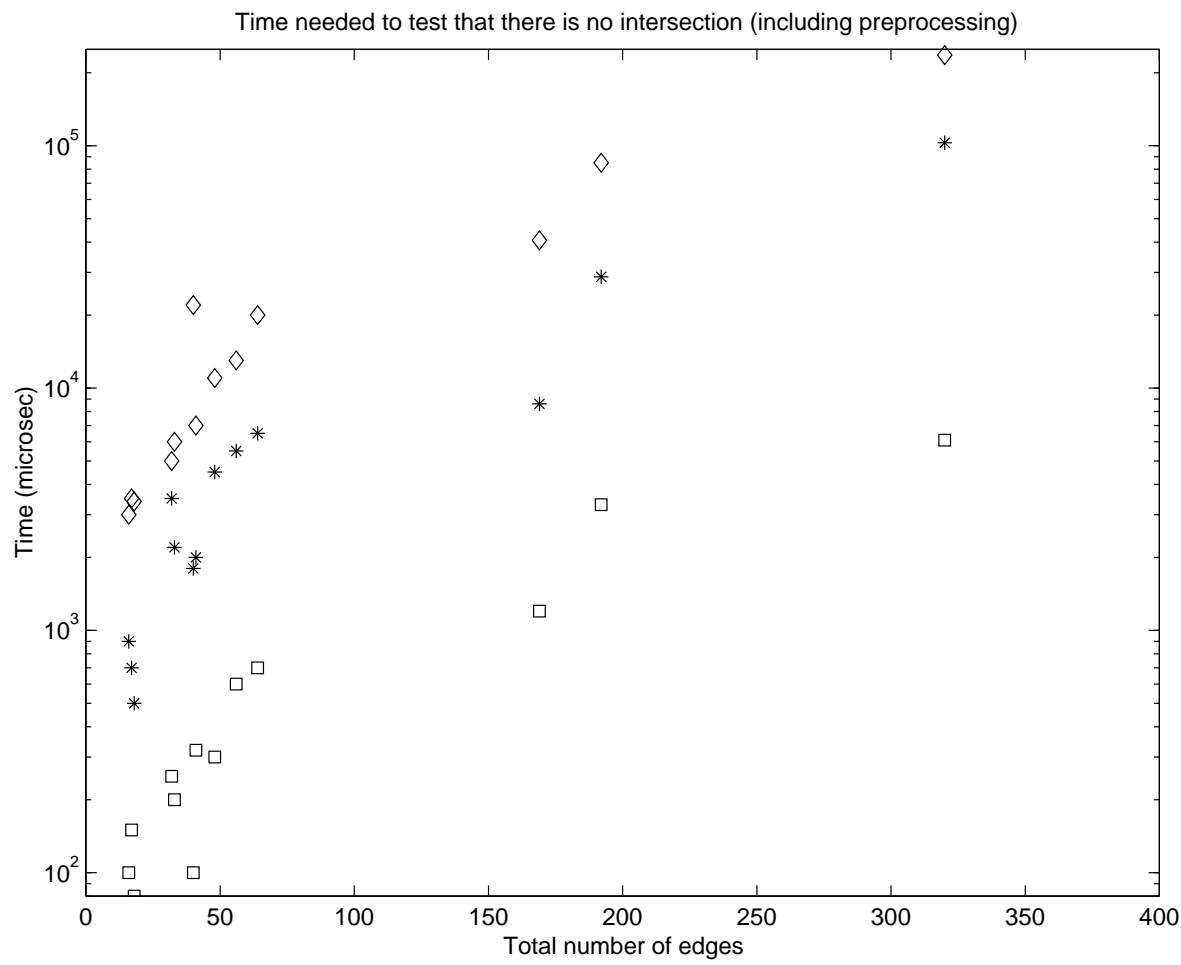

Figure 19: Comparison of the execution times of algorithm $A\left(^{*}\right)$, and algorithm $C(\square)$, with a previous pruning step ( $\diamond)$, in settings that include only nonconvex polyhedra.

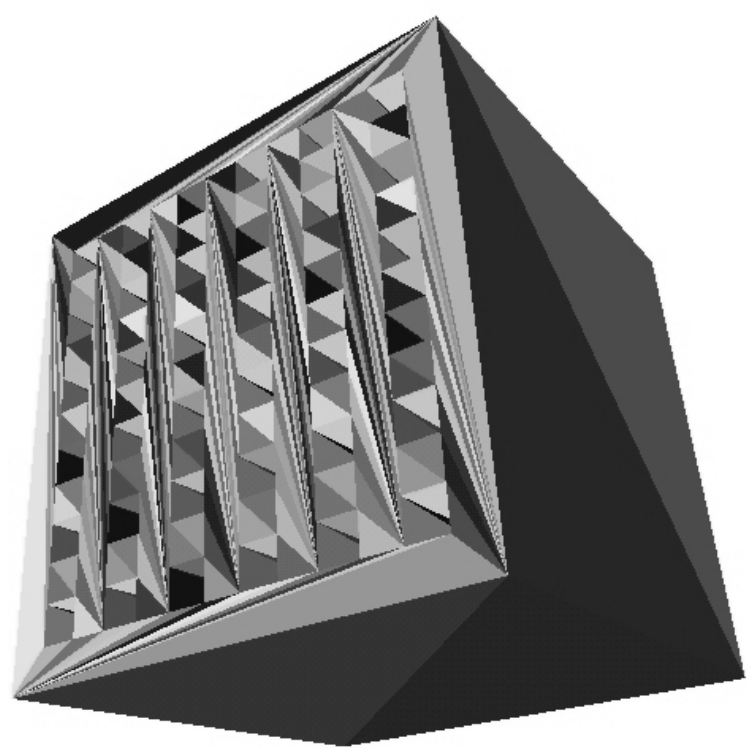

Figure 20: The type of workpiece used to compare the performances of algorithm $C$ and RAPID. The faces of the polyhedron (displayed here for the case of $6 x 6$ holes) have been triangulated with an implementation of Seidel's algorithm (the code has been obtained at http://www.cs.unc.edu/ ${ }^{\sim} \mathrm{dm} / \mathrm{CODE} /$ GEM/chapter.html). Remember that triangulation is a necessary previous step for RAPID, but not for our algorithm. 


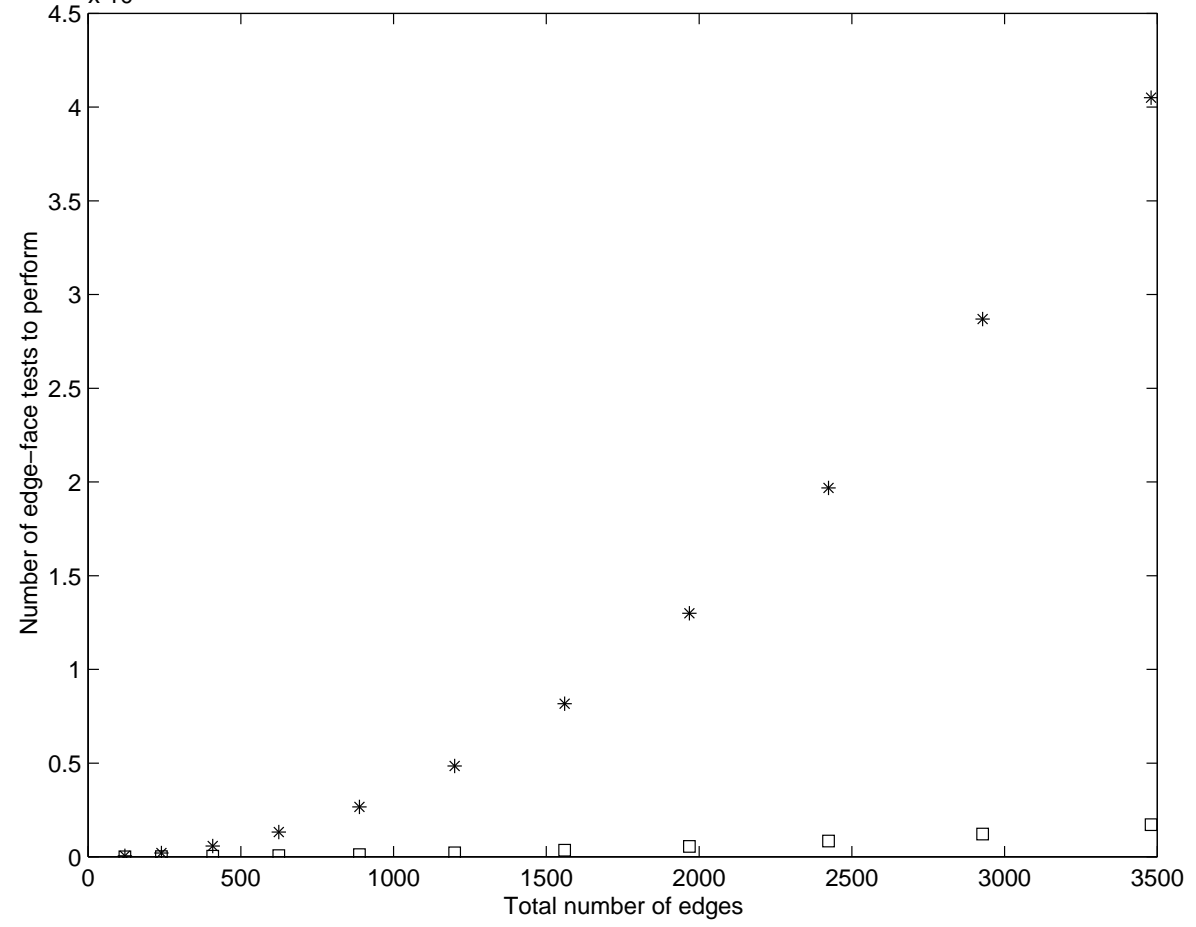

Figure 21: Number of edge-face intersection tests to perform by algorithm $C$ ( $\square$ ) against the total number of edge-face pairings $\left({ }^{*}\right)$, for the setting with two cubes having an increasing number of holes.

the case of two cubes with $12 \times 12$ holes each (e.g., having 3480 edges), algorithm $\mathrm{C}$ detects all contacts in $620 \mathrm{~ms}$, while RAPID needs $3051 \mathrm{~ms}$. The gain in speed is attained at the expense of a much higher preprocessing time (Figure 23). In the latter figure, times that are accounted for are searching the SFOG representation for determining all pairs of applicable features and extracting all possibly intersecting edgeface pairings, for algorithm C; whereas for RAPID the measured time corresponds to Seidel's triangulation plus building up the hierarchical data structures.

It should be noted that the comparison with RAPID obeys exclusively the purpose of showing the potential of our approach. Thus, we have chosen the most favourable setting for our algorithm, namely two objects with many concavities, placed in a way that many simultaneous contacts occur. Both algorithms are in some way complementary, in the sense that pruning is based on volumetric aspects in RAPID, while the algorithm presented here exploits orientation information. Thus, both approaches are not mutually exclusive, and possibilities exist for their integration.

\section{Conclusions}

An orientation-based pruning strategy for reducing the computational effort in contact determination between general polyhedra has been described. This strategy is based on applicability conditions and consists basically in reducing the number of edge-face pairs to be considered for intersection, by taking into account that only a subset of all contacts between the features of two polyhedra is actually possible, if the relative orientation of the polyhedra does not change. It has to be stressed that, in our approach, nonconvex polyhedra can be directly tested for interference, without decomposing them into convex entities. Therefore, applicability pruning is a preprocessing step, like the usual practice of decomposing the polyhedra into convex entities, but it does not increase the complexity of the setting by introducing fictitious features, as the convex decomposition does.

Experiments carried out in settings with convex and nonconvex polyhedra show important savings in the computational effort: contact determination based on the edge-face intersection test performs 10 to 100 times faster if a previous selection of candidate pairs based on the applicability conditions is performed. Although highly dependent on the specific geometry of the involved polyhedra, it can also be stated that these savings increase proportionally to the complexity of the setting.

Our approach used alone, without a bounding volume hierarchy or any other encapsulation method, can outperform interference detection packages such as RAPID only under very restrictive conditions, 


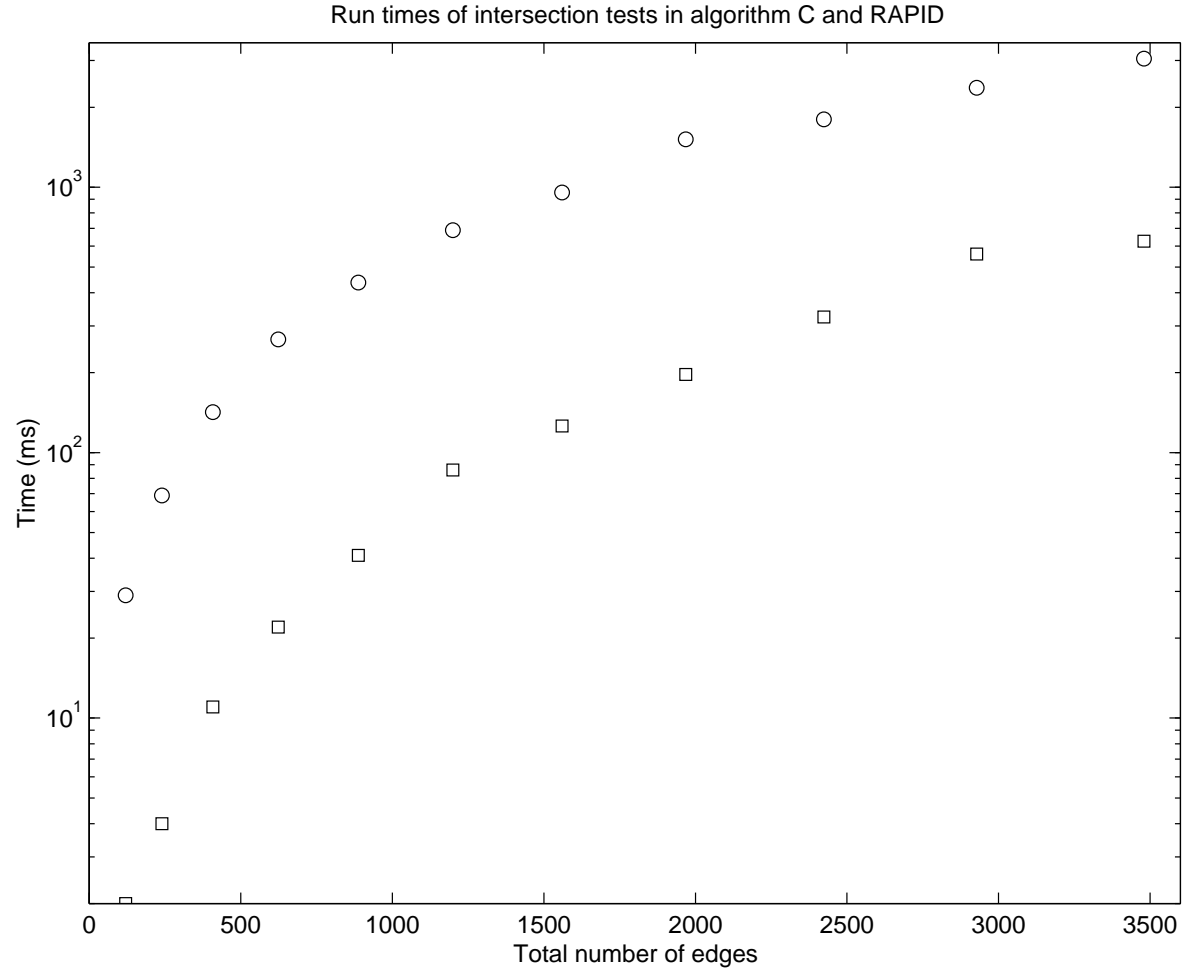

Figure 22: Comparison of the execution times of the interference detection phase in algorithm $C(\square)$ and RAPID (०).

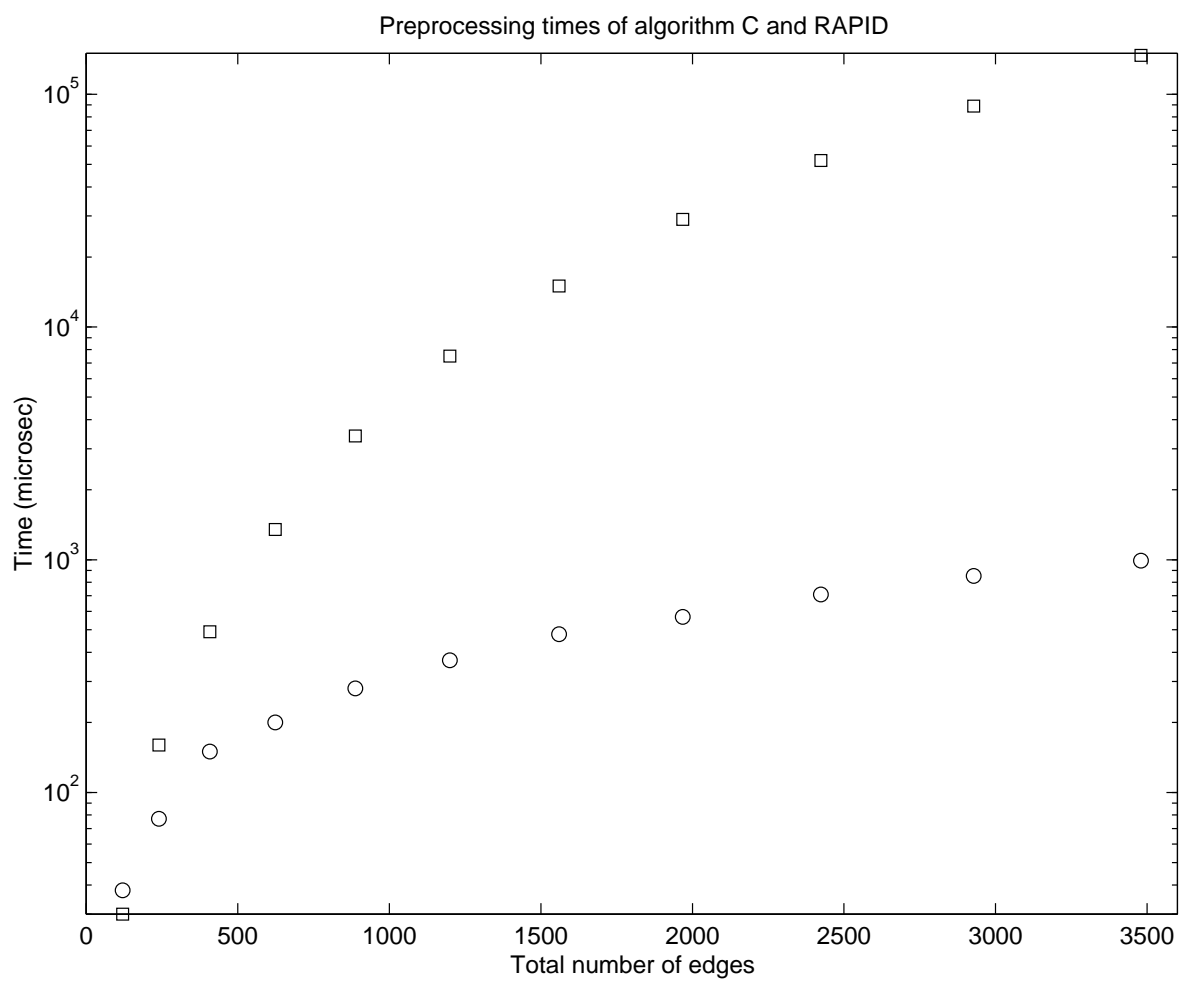

Figure 23: Comparison of preprocessing times of algorithm $C$ ( $\square$ ) and RAPID (०). Preprocessing is various orders of magnitude slower for the former. However, it has to be performed only once in settings where objects are only allowed to translate. 
may simultaneously occur. This is a typical situation arising in assembly design and planning within CAD/CAM systems.

Moreover, both the edge-face intersection test and the proposed orientation-based pruning can be seen as general tools, which can be combined with other space and time bounding strategies, and integrated in a given collision detection scheme.

Two issues deserve further attention. First, since many computer graphics applications depend on surface normals, several data structures have been proposed for encapsulating surface orientations. Most of them were developed to tackle interactions between a point and a polyhedral model, but recently one such structure, namely the spatialized normal cone hierarchy, has been applied in the computation of local distances in polyhedral settings [Johnson and Cohen, 2001]. We see this as the orientation-based counterpart effort to that of establishing hierarchies of bounding volumes and, therefore, we would like to explore the possibility of integrating such type of hierarchy into our approach.

The other issue is that the applicability conditions are orientation-dependent, which means that along trajectories that entail a change in the relative orientation of the polyhedra, new edge - face candidates arise, while others become no longer valid. Therefore, one must be able to determine the intervals of isoapplicability, i.e., the ranges of relative orientations -along the trajectory- for which the same applicability conditions hold. In a trajectory parameterization approach, this means to determine the values of the parameter where these changes occur, whereas in a multiple interference detection approach a discretization of time based on isoapplicability will have to be considered, besides the standard discretization based on distance and relative velocities. The SFOG representation can be used to this end: the intervals of isoapplicability are delimited by the rotation events, each time a node of one SFOG crosses an arc of the other one. These questions are addressed in [Jiménez, 1998], but devising an efficient method for computing all the rotation events for arbitrary changes in the relative orientation of the polyhedra is still an open issue.

\section{ACKNOWLEDGEMENTS}

This research has been partially supported by the Spanish Science and Technology Commission (CICYT) under contract TAP99-1086-C03-01 (project "Constraint-based computation in robotics and resource allocation") and the Catalan Research Commission, through the "Robotics and Control" group. We would like to thank two anonymous reviewers for their helpful comments.

\section{REFERENCES}

\section{References}

[Agarwal, 1990] Agarwal, P. (1990). Partitioning arrangements of lines. ii: Applications. Discrete Comput. Geom., 5:533-573.

[Agarwal and Sharir, 1990] Agarwal, P. and Sharir, M. (1990). Red-blue intersection detection algorithms, with applications to motion planning and collision detection. Siam J. Comput., 19(2):297-321.

[Basch et al., 1996] Basch, J., Guibas, L. J., and Ramkumar, G. D. (1996). Reporting red-blue intersections between connected sets of line segments. In 4th European Symposium on Algorithms, pages 302-319.

[Boyse, 1979] Boyse, J. W. (1979). Interference detection among solids and surfaces. Comm. ACM, $22(1): 3-9$.

[Cameron, 1991] Cameron, S. A. (1991). Efficient bounds in constructive solid geometry. IEEE Computer Graphics and Applications, pages 68-74.

[Canny, 1987] Canny, J. (1987). The Complexity of Robot Motion Planning. MIT Press, Cambridge, MA.

[Cohen et al., 1995] Cohen, J. D., Lin, M. C., Manocha, D., and Ponamgi, M. K. (1995). I-collide: An interactive and exact collision detection system for large-scale environments. In Proceedings of ACM Int. 3D Graphics Conference, volume 1, pages 189-196. http://www.cs.unc.edu / geom /I_COLLIDE.html.

[Donald, 1987] Donald, B. R. (1987). A search algorithm for motion planning with six degrees of freedom. Artificial Intelligence, 31(3):295-353. 
detection problem. IEEE Computer Graphics and Applications, 14(3):36-43.

[Gilbert et al., 1988] Gilbert, E. G., Johnson, D. W., and Keerthi, S. (1988). A fast procedure for computing the distance between complex objects in three dimensional space. IEEE J. Robotics Automat., $4(2): 193-203$.

[Gottschalk et al., 1996] Gottschalk, S., Lin, M. C., and Manocha, D. (1996). Obb-tree: A hierarchical structure for rapid interference detection. In Proc. of ACM Siggraph'96. http://www.cs.unc.edu / geom/OBB /OBBT.html.

[Hamlin et al., 1992] Hamlin, G. J., Kelley, R. B., and Tornero, J. (1992). Efficient distance calculation using the spherically-extended polytope (s-tope) model. In Proc. IEEE Int. Conf. on Robotics and Automation, volume 3, pages 2502-2507, Nice (France).

[Hilbert and S.Cohn-Vossen, 1987] Hilbert, D. and S.Cohn-Vossen (1987). Geometry and the Imagination. Chelsea.

[Horn, 1984] Horn, B. K. (1984). Extended gaussian images. In Proc. of the IEEE, volume 72, pages $1671-1686$.

[Hubbard, 1993] Hubbard, P. M. (1993). Interactive collision detection. In Proc. IEEE Symp. on Research Frontiers in Virtual Reality, volume 1, pages 24-31.

[Hubbard, 1995] Hubbard, P. M. (1995). Collision detection for interactive graphics applications. IEEE Transactions on Visualization and Computer Graphics, 1(3):218-230.

[Hudson et al., 1997] Hudson, T. C., Lin, M. C., Cohen, J. D., Gottschalk, S., and Manocha, D. (1997). V-collide: Accelerated collision detection for vrml. In Proceedings of VRML. http://www.cs.unc.edu/ geom/V_COLLIDE .html.

[Jiménez, 1998] Jiménez, P. (1998). Static and dynamic interference detection between nonconvex polyhedra. $\mathrm{PhD}$ thesis, Universitat Politècnica de Catalunya. http://wwwiri.upc.es/people/jimenez/phdthesis.html.

[Jiménez et al., 2001] Jiménez, P., Thomas, F., and Torras, C. (2001). Collision detection: a survey. Computers and Graphics, 25(2):269-285.

[Jiménez and Torras, 1996] Jiménez, P. and Torras, C. (1996). Speeding up interference detection between polyhedra. In Proc. IEEE Int. Conf. on Robotics and Automation, volume 2, pages 1485-1492, Minneapolis (MN).

[Jiménez and Torras, 1999] Jiménez, P. and Torras, C. (1999). Benefits of applicability constraints in decomposition-free interference detection between nonconvex polyhedral models. In Proc. IEEE Int. Conf. on Robotics and Automation, volume 3, pages 1856-1862, Detroit (MI).

[Jiménez and Torras, 2000] Jiménez, P. and Torras, C. (2000). An efficient algorithm for searching implicit and/or graphs with cycles. Artificial Intelligence, 124(1):1-30.

[Johnson and Cohen, 2001] Johnson, D. and Cohen, E. (2001). Spatialized normal cone hierarchies. In Proc. 2001 ACM Symposium on Interactive 3D Graphics, volume 2, pages 129-134, Research Triangle Park, (NC).

[Klosowski et al., 1998] Klosowski, J., Held, M., Mitchell, J., Sowizral, H., and Zikan, K. (1998). Efficient collision detection using bounding volume hierarchies of k-dops. IEEE Transactions on Visualization and Computer Graphics, 4(1).

[Lin and Canny, 1991] Lin, M. C. and Canny, J. F. (1991). A fast algorithm for incremental distance calculation. In Proc. IEEE Int. Conf. on Robotics and Automation, volume 2, pages 1008-1014, Sacramento $(\mathrm{CA})$.

[Lin and Gottschalk, 1998] Lin, M. C. and Gottschalk, S. (1998). Collision detection between geometric models: a survey. In IMA Conference on Mathematics of Surfaces, volume 1, pages 602-608, San Diego $(\mathrm{CA})$. 
for practical motion planning. part i: The spatial representation. In Proc. IEEE Int. Conf. on Robotics and Automation, volume 1, pages 624-629, Leuven (Belgium).

[Pearl, 1984] Pearl, J. (1984). Heuristics: Intelligent Search Strategies for Computer Problem Solving. Addison-Wesley.

[Ponamgi et al., 1997] Ponamgi, M. K., Manocha, D., and Lin, M. C. (1997). Incremental algorithms for collision detection between polygonal models. IEEE Trans. on Visualization and Comp. Graphics, $3(1): 51-64$.

[Preparata and Shamos, 1985] Preparata, F. F. and Shamos, M. I. (1985). Computational Geometry: An Introduction. Texts and Monographs in Computer Science. Springer Verlag, New York.

[Thibault and Naylor, 1987] Thibault, W. C. and Naylor, B. F. (1987). Set operations on polyhedra using binary space partitioning trees. ACM Computer Graphics, 21(4).

[Thomas and Torras, 1992] Thomas, F. and Torras, C. (1992). Inferring feasible assemblies from spatial constraints. IEEE Trans. on Robotics and Automation, 8(2):228-239.

[Thomas and Torras, 1994] Thomas, F. and Torras, C. (1994). Interference detection between non-convex polyhedra revisited with a practical aim. In Proc. IEEE Int. Conf. on Robotics and Automation, volume 1, pages 587-594, San Diego (CA).

[van der Bergen, 1997] van der Bergen, G. (1997). Efficient collision detection of complex deformable models using aabb trees. Journal of Graphic Tools, 2(4):1-13.

[Vanecek, 1994] Vanecek, G. (1994). Back-face culling applied to collision detection of polyhedra. Journal of Visualization and Computer Animation, 5(1):55-63. 